\title{
Electrocardiographic changes following intracranial haemorrhage: a retrospective cohort study
}

\author{
Bassam E. Yaghmoor, MBBS, Shayma M. Alotaibi, MBBS, Maryam Z. Enani, MBBS, \\ Hussam S. AlQudsi, MBBS, Masaheer A. Aljehani, MBBS, Mshari H. Althomali, MBBS, Fatimah M. Hisan, MBBS, \\ Ghaidaa J. Sindi, MBBS, Naeem A. Alshoaibi, MBBS, FRCP, Abdulrahman J. Sabbagh, MBChB, FRCS.
}

\begin{abstract}

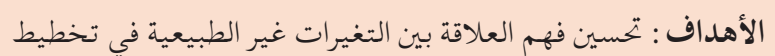

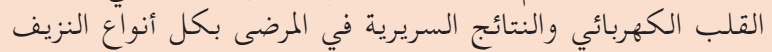
داخل الرأس.

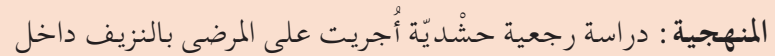

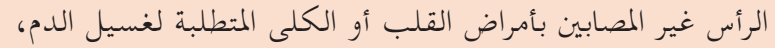

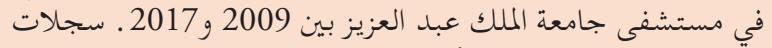

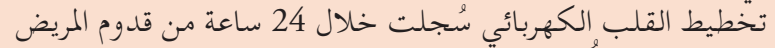

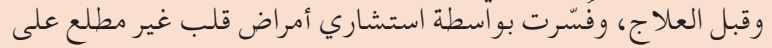
البيانات الأخرى.

النتائج : تقييم 291 مريضًا ( 228 بالغين و63 أطفال ) أظهر أن أن أنساف

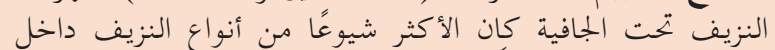

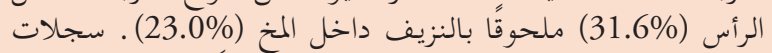

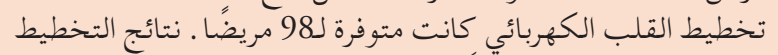

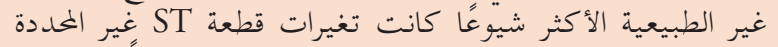

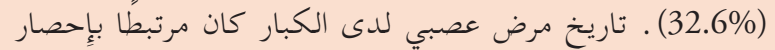

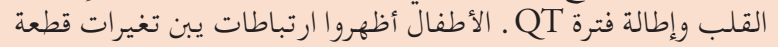

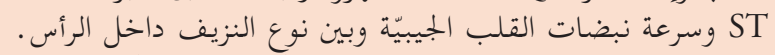

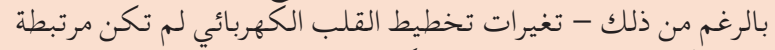
إحصائيًا بالنتائج السريرية في الكبار أو الأطفال . لخطيات

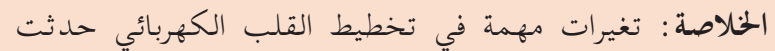

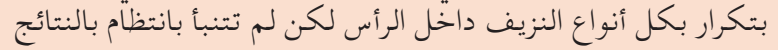

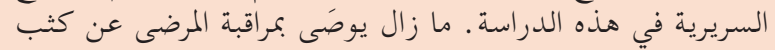

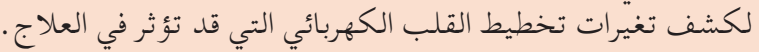

Objectives: To improve the understanding of the association between electrocardiographic (ECG) abnormalities and clinical outcomes of patients with all types of intracranial hemorrhage (ICH).

Methods: A retrospective cohort study was conducted in a tertiary healthcare hospital on patients with ICH without cardiac disease or renal disease requiring dialysis. Demographic and clinical data were collected from hospital records. ECG record were obtained within 24 hours of presentation and prior to treatment. Records were interpreted for this study by a cardiologist blinded to other data.

Results: Assessment of 291 patients (228 adults and 63 children) showed that subdural hemorrhage was the most common type of ICH (31.6\%) followed by intraparenchymal hemorrhage (23.0\%). ECG records were available for 98 patients. ECG abnormalities were most commonly nonspecific ST-segment changes $(32.6 \%)$. In adults, history of neurological disease was associated with atrioventricular block $(p=.004)$ and QTc prolongation $(p=.041)$. Pediatric patients exhibited associations between ST-segment changes $(p=.045)$ and sinus tachycardia $(p=.027)$ and type of ICH. However, ECG changes were not statistically associated with clinical outcomes in adults or children.

Conclusion: Significant ECG changes frequently occurred in patients with all types of ICH but did not consistently predict the outcome in this study. Close observation of patients is still recommended to detect ECG changes that could affect the treatment.

\section{Neurosciences 2020; Vol. 25 (2): 104-111 doi: 10.17712/nsj.2020.2.20190109}

From the Faculty of Medicine (Yaghmoor, Alotaibi, Enani, AlQudsi, Aljehani, Althomali, Hisan, Sindi), Division of Cardiology (Alshoaibi), Department of Internal Medicine, and from the Division of Neurosurgery (Sabbagh), Department of General Surgery, Faculty of Medicine, King Abdulaziz University, Jeddah, Kingdom of Saudi Arabia

Received 9th November 2019. Accepted 19th December 2019.

Address correspondence and reprint request to: Dr. Bassam E. Yaghmoor, Faculty of Medicine, King Abdulaziz University, Jeddah, Kingdom of Saudi Arabia.E-mail:bas_yag@hotmail.com

ORCID ID: https://orcid.org/0000-0003-2775-0170

Tntracranial hemorrhages (ICHs) are classified, 1 depending on the cause, to traumatic and nontraumatic. ${ }^{1,2}$ Anatomical site further classifies ICHs into 4 main groups. ${ }^{1}$ Epidural hemorrhage $(\mathrm{EDH})$ is 
defined as the blood collection between the bone and the dura mater. ${ }^{1}$ Subdural hemorrhages $(\mathrm{SDH})$ are defined as the collection of blood beneath the dura and above the arachnoid membrane. ${ }^{1}$ Subarachnoid hemorrhages (SAH) are defined as bleeding into the space beneath the arachnoid membrane and above the pia mater. ${ }^{1}$ It commonly occurs after rupture of an aneurysm or an arteriovenous malformations in the brain. ${ }^{3}$ Non-traumatic, or spontaneous, ICHs usually involve the brain parenchyma and the subarachnoid space. $^{2}$ Non-traumatic intraparenchymal hemorrhages (IPH) result from bleeding of small arteries, commonly due to hypertension; IPHs account for $10-20 \%$ of all strokes cases. ${ }^{4}$ Non-traumatic hemorrhages resulting from other causes are possible, such as hemorrhage associated with brain tumors and infections. It is well known that ICHs are associated with high mortality and morbidity rates on their own, but neurocardiac injuries following cerebrovascular events such as subarachnoid and cerebral hemorrhages worsen the clinical outcomes even more. ${ }^{5}$

Stress on the nervous system can affect the heart functionally and structurally, a phenomenon known as neurogenic stress cardiomyopathy (NSC). The NSC occurs frequently in some types of ICHs such as non-traumatic $\mathrm{SAH}$ and non-traumatic IPH. ${ }^{6-8}$ Electrocardiographic (ECG) changes occur in $49 \%$ to $100 \%$ of patients following SAH. ${ }^{9,10}$ The most common ECG alterations following SAH and IPH are repolarization abnormalities such as QT interval prolongation, ST segment and T wave changes. ${ }^{11,12}$ Atrioventricular (AV) block, atrial flutter, and ventricular arrhythmia are the most common alterations associated with cardiac arrhythmias ${ }^{13}$ although the mechanisms are not yet fully uncovered. The catecholamine hypothesis, also known as catecholamine-mediated direct cardiac injury, is the most widely accepted proposed mechanism underlying the manifestation of NSC, whereby catecholamines directly damage cardiac tissue. $^{6,14}$ The histopathological finding associated with excess catecholamine is myocardial contract band necrosis. ${ }^{15}$ A study by Naredi et $\mathrm{al}^{14}$ reported elevated levels of catecholamines in cases of $\mathrm{SAH}$.

The NSC may also be caused by traumatic brain injury though it has only been documented in a few case series, and no clear incidence has been reported.

Disclosure. Authors have no conflict of interests, and the work was not supported or funded by any drug company.
The standard biomarker used to diagnose NSC is serum troponin 1, which has a sensitivity of $100 \%$ and specificity of $91 ;^{6}$ elevated levels are associated with poor prognosis. ${ }^{13}$ New onset of ECG abnormalities have been found in $75 \%$ of patients following an acute cerebrovascular event without any previous history of heart disease. ${ }^{13}$ ECG abnormalities are associated with higher risk of poor outcomes in patients with NSC. ${ }^{5,7,8}$

This study aimed to improve our understanding of the possible association between ECG abnormalities, different types of $\mathrm{ICHs}$, and radiological findings. Furthermore, it aimed to identify the risk and prognostic factors associated with neurocardiac injury, which can be beneficial in identifying vulnerable patients and predicting possible adverse events.

Methods. Study design \& parameters. This retrospective cohort study was conducted in a tertiary healthcare hospital and approved by the Research Ethics Committee and is in line with the principles of Helsinki Declaration. Considering the nature of this study, informed consent was not required.

Search for prior related studies was done through PubMed database using the keywords "intracranial hemorrhage", "epidural hemorrhage", "subdural hemorrhage", "subarachnoid hemorrhage", "intracerebral hemorrhage", "electrocardiographic changes", "neurocardiac injury", and "outcome", separate and in combinations. Systematic reviews and meta analyses were prioritized when found, and studies as new as 2014 or newer were favored.

This study was performed by reviewing the medical records of 384 patients diagnosed with ICH, admitted to the hospital between 2009 and 2017; the diagnosis was confirmed by computed tomography (CT) scans.

All cases of traumatic and non-traumatic $\mathrm{ICH}$ were included in this study. Ninety-two patients were excluded from the study either due to unavailable clinical or radiological findings or due to a known history of heart disease, cardiac arrhythmia, or end-stage renal disease requiring dialysis. Cases of $\mathrm{ICH}$ related to infectious or oncological causes were excluded. Therefore, the statistical analysis included 292 patients in total; patients aged $0-18$ accounted for 63 of the cases, and patients aged $>18$ years accounted for 229 cases.

Data collected for analysis included demographic information, clinical presentations, laboratory results, and radiological data, including vital signs, electrolyte levels, concentration of serum creatinine, and blood urea nitrogen (BUN), all of which were recorded at the time of presentation. Other data associated with pathological 
findings including skull fractures, hydrocephalus, midline shift, neurogenic pulmonary edema, delayed cerebral ischemia, and hospital-acquired infections were also recorded. Level of consciousness was assessed by the Glasgow Coma Scale (GCS), which was documented at the time of presentation and before discharge or death. The site of hematoma was categorized as right or left hemisphere, or bilateral. Hematoma volume was calculated in cubic centimeters $\left(\mathrm{cm}^{3}\right)$. Fisher grading scale and Hunt and Hess grading scale were utilized to predict the mortality and morbidity rates, vasospasm risk, and outcome in patients with $\mathrm{SAH}$, for which a higher grade predicts a poorer outcome and a lower likelihood of survival. Patient outcome and the degree of neurological disability of patients recovering from ICH were measured by the Modified Rankin Scale (MRS) and length of stay until discharge or death.

Electrocardiogram analysis. The ECG (12-lead) data were collected within 24 hours of patient presentation and before treatment and was obtained at a paper speed of 25 millimeters per second, with an amplitude calibration of 10 millimeters per millivolt. All ECGs were analyzed by an experienced cardiologist blinded to clinical and follow-up data. The ECG changes were documented if the abnormality was noted in at least 2 leads and were classified according to the modified Minnesota Code. ${ }^{16}$ ECG changes were assessed and classified based on the following parameters: (1) Rhythm: sinus rhythm, atrial fibrillation, or atrial flutter. (2) Rate: sinus bradycardia (less than 60 beats per minute) or sinus tachycardia (more than 100 beats per minute). (3) PR interval: normal (0.12 to 0.21 seconds) or prolonged (more than 0.21 seconds). (4) QRS complex width: prolonged if equal to or more than 0.12 seconds. (5) QTc prolongation (equal to or more than 440 milliseconds in men and 460 milliseconds in women). (6) Morphology (P wave, QRS complex, ST segment, T wave, Q wave, and U wave). (7) ST segment depression: down sloping or horizontal (more than 0.05 millivolts). (8) ST segment elevation: convexity of the ST segment upwards (more than 0.1 millivolts). (9) Prominent $U$ wave: more than $25 \%$ of the largest $\mathrm{T}$ wave amplitude in precordial leads.

Statistical analysis. All of the values were expressed as number (\%) or mean \pm standard deviation (SD), as appropriate. Data were analyzed using Statistical Package for Social Sciences (SPSS Inc., version 22, Chicago, IL, USA). The data were analyzed by the Chi-Square test to compare between categorical variables and interdependent T-test or one-way analysis of variance (ANOVA) when involving numerical variables. A $p$-value less than or equal to 0.05 was considered statistically significant, with a $95 \%$ confidence interval. Missing data were dropped from the analysis.

Results. The study included 291 participants with an average age of $59 \pm 34$ years. Sixty-three (21.6\%) participants were included in the pediatric group (below 18 years of age); 228 (78.4\%) participants were included in the adult group (above 18 years of age). Two hundred and six $(70.8 \%)$ participants were males; only $86(29.2 \%)$ participants were females.

Of the 291 research participants, $152(52.2 \%) \mathrm{ICH}$ cases were traumatic, while $140(47.8 \%)$ cases were non-traumatic in origin. Overall, $\mathrm{SDH}$ was the most type of $\mathrm{ICH}$. The death rate of the patients in this study was $18.9 \%$. Although $76.3 \%$ of the participants were discharged from the hospital, they experienced complications such as hydrocephalus (10.7\%), midline shift $(7.6 \%)$, and intraventricular hemorrhage (IVH) (7.9\%). One percent were in a vegetative state.

Among the 291 study participants, ECGs were only available for 98 cases. Abnormalities were identified in $62.2 \%$ of the ECGs. Fifty percent of patients with neurological diseases for whom ECG records were available had 1st or 3rd degree AV blocks, which is significantly higher than those without neurological disease $(p=.004)$. There were 4 patients with neurological diseases and ECG records; 3 had strokes, one of whom also had Alzheimer's disease with 3rd degree AV block, and one with only trigeminal neuralgia with 1 st degree AV block. All of them were adults. QTc prolongation was related to these neurological conditions $(25 \%$ of those with neurological disease, trigeminal neuralgia vs $0 \%$ of those without QTc prolongation) ( $p=.041)$.

Nevertheless, none of the ECG abnormalities were significantly associated with ICH outcomes ( $p>.05)$.

Sociodemographic dimensions of the adult ICH group. The subcategory "Adult ICH" was comprised of 228 participants with an average age of $56 \pm 19$ years. One hundred and sixty-seven $(73.2 \%)$ cases were male, whereas $61(26.8 \%)$ were female. The prevalence of each ICH type with a traumatic or non-traumatic origin is shown in Table 1.

ECG changes in the adult ICH group. Out of 228 adult participants, ECG results were available for only 87 adults.

1. Prevalence of ECG Abnormalities in the Adult ICH Group. Abnormalities were identified in $60.9 \%$ of the ECGs. ECG abnormalities in the Adult ICH Group are displayed in Table 2.

2. Associations of ECG Abnormalities and clinical outcomes in the Adult ICH Group. Abnormal 


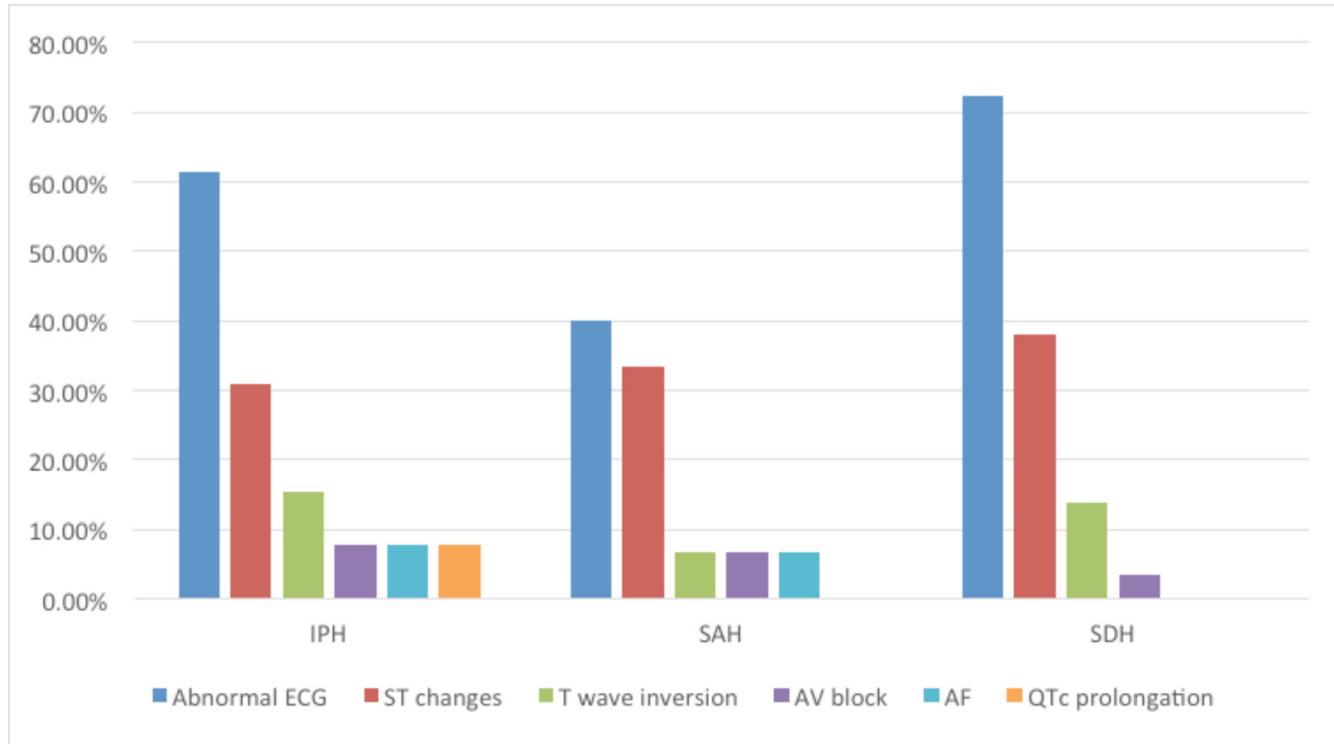

Figure 1 - Prevalence of some electrocardiographic (ECG) abnormalities in different types of Adult ICH. It shows that most ECG records in intraparenchymal and subdural hemorrhages were abnormal with ST-segment changes being the most common, while in subarachnoid hemorrhage, less than have the records were abnormal but ST-segment changes were still predominating. *IPH - intraparenchymal hemorrhage, SAH - subarachnoid, $\mathrm{SDH}$ - subdural hemorrhag

ECG was significantly related to age group $(p=.032)$. Of the patients 60 years of age or older $(\mathrm{n}=97), 79 \%$ had abnormal ECGs compared to $49 \%$ and $52 \%$ of patients aged 40-60 ( $\mathrm{n}=81)$ and below 40 years old $(\mathrm{n}=50)$, respectively. Similarly, LAD was statistically related to age group ( $p=.017) ; 24.2 \%$ of patients 60 years or older had LAD compared to $6.1 \%$ and $0 \%$ of patients $40-60$ and below 40 years old, respectively.

The AV block was significantly associated $(p=.005)$ with neurological diseases ( 2 of 4 patients with neurological disease; one had 1st degree AV block and trigeminal neuralgia and the other had 3rd degree AV block and Alzheimer's disease). Also, QTc prolongation

Table 1 - Prevalence of ICH types of traumatic or non-traumatic origin in the 'Adult ICH Group'. Nineteen cases were missing information about being traumatic or non-traumatic.

\begin{tabular}{lcc}
\hline Type of ICH & $\begin{array}{c}\text { Traumatic } \\
(\mathbf{n}=107)\end{array}$ & $\begin{array}{c}\text { Non-traumatic } \\
(\mathbf{n}=102)\end{array}$ \\
\hline Intraparenchymal & - & $(54.9)$ \\
Subarachnoid & $(9.3)$ & $(30.4)$ \\
Subdural & $(62.6)$ & - \\
Epidural & $(10.3)$ & - \\
Intraventricular & $(0.9)$ & $(2)$ \\
Mixed & $(16.8)$ & $(12.7)$ \\
\hline \multicolumn{2}{c}{} \\
\hline
\end{tabular}

remained significantly related to neurological diseases (one of the 4 patients with neurological disease; they had trigeminal neuralgia, $p=.046$ ). Nevertheless, none of the ECG findings were statistically associated with ICH clinical outcomes (Table 3) ( $p>.05$ ).

3. ECG Results in the Adult IPH, Adult SAH and Adult SDH subclasses. Thirteen ECGs were available for the adult patients with intraparenchymal hemorrhages, 15 ECGs were available for the adult patients with subarachnoid hemorrhages, and 29 ECGs were available for the adult patients with subdural hemorrhages. The prevalence of some of the ECG abnormalities are plotted for each of the 3 adult subgroups in Figure 1; none were statistically significant $(p>.05)$. Among adult $\mathrm{SDH}$ patients, those who suffered from neurological diseases had a significant association with 3rd degree AV block ( $p=.034)$.

Socio-Demographic Dimensions of Pediatric ICH Group. The subcategory "Pediatric ICH" was comprised of 63 participants of an average age of $6 \pm 5.6$ years. Thirty-nine $(61.9 \%)$ of the children were males; $24(38.1 \%)$ were females. Forty-five cases $(71.4 \%)$ were traumatic and $18(28.6 \%)$ were non-traumatic in origin. Prevalence of each type of $\mathrm{ICH}$ is shown in Table 4. Discharge and death prevalence of traumatic ICH in the pediatric subgroup were $88.9 \%$ and $11.1 \%$ respectively. Nearly $9 \%$ of the traumatic $\mathrm{ICH}$ victims 
Table 2 - Prevalence of electrocardiographic (ECG) Abnormalities in the Adult ICH.

\begin{tabular}{lc}
\hline ECG Abnormality & $(\%)$ \\
\hline ST Changes & $(32.2)$ \\
T Inversion & $(13.8)$ \\
AV Block & $(3.5)$ \\
Right bundle branch block & $(3.5)$ \\
Left bundle branch block & $(1.2)$ \\
Left axis deviation & $(11.5)$ \\
Atrial fibrillation & $(3.5)$ \\
Q Wave & $(2.3)$ \\
QTc Prolongation & $(1.2)$ \\
\hline AV - Atrioventricular, ICH - Intracranial hemorrhage
\end{tabular}

Table 3 - Crosstabulation of ECG results and ICH outcomes in adults. Data were analyzed by a Chi-squared test.

\begin{tabular}{|c|c|c|c|}
\hline \multirow[t]{2}{*}{ ECG result } & \multicolumn{2}{|c|}{$\begin{array}{l}\text { Final outcome } \\
n(\%)\end{array}$} & \multirow[t]{2}{*}{$P$-value } \\
\hline & Death & Discharge & \\
\hline Normal & $6(18.2)$ & $27(81.8)$ & \multirow{2}{*}{.616} \\
\hline Abnormal & $6(11.8)$ & $45(88.2)$ & \\
\hline
\end{tabular}

developed hydrocephalus afterwards. Discharge and death prevalence of non-traumatic $\mathrm{ICH}$ in the pediatric subgroup were $66.7 \%$ and $33.3 \%$, respectively.

ECG Changes in the Pediatric ICH Group. Out of 63 pediatric ICH patients, ECG results were only available for 11 children.

1. Prevalence of ECG Abnormalities in the Pediatric ICH Group. Abnormalities were identified in $72.7 \%$ of the ECGs. ECG abnormalities in the 'Pediatric ICH Group' are shown in Table 5.

2. Associations between ECG Abnormalities and clinical outcomes in the Pediatric ICH Group. The ST changes were noted in 50\% of intraparenchymal and $100 \%$ of both SAH and spontaneous mixed ICH. ST changes were significantly related to the type of $\mathrm{ICH}$ $(p=.045)$.

One hundred percent of both EDH and spontaneous mixed ICH were reported to have tachycardia. Therefore, tachycardia was statistically related to the type of $\mathrm{ICH}$ $(p=.027)$. Nevertheless, none of the ECG findings were statistically associated with ICH outcomes ( $p>.05)$.

Discussion. In our study, $52.2 \%$ of patients had traumatic ICH, while $47.8 \%$ of cases were of nontraumatic origin. The most frequent type $\mathrm{ICH}$ was $\mathrm{SDH}$ (31.6\% of cases). Death was prevalent in $18.9 \%$ of all cases. The ECG records for 98 patients were available, of which $62.2 \%$ were abnormal.
Table 4 - Prevalence of different ICH types in the 'Pediatric ICH Group'.

\begin{tabular}{lc}
\hline Type of ICH & $\mathbf{n}(\%) \mathbf{N}=\mathbf{6 3}$ \\
\hline Intraparenchymal & $(12.7)$ \\
Subarachnoid & $(6.3)$ \\
Subdural & $(39.7)$ \\
Epidural & $(20.6)$ \\
Intraventricular & $(1.6)$ \\
Mixed & $(19.1)$ \\
\hline \multicolumn{2}{c}{ ICH - Intracranial hemorrhage } \\
\hline
\end{tabular}

Table 5 - Prevalence of electrocardiographic (ECG) abnormalities in the Pediatric ICH Group.

\begin{tabular}{lc}
\hline ECG Abnormality & $(\%)$ \\
\hline ST Changes & $(36.4)$ \\
T Inversion & $(36.4)$ \\
AV Block & $(0)$ \\
Right bundle branch block & $(9.1)$ \\
Left bundle branch block & $(0)$ \\
Left axis deviation & $(0)$ \\
Atrial fibrillation & $(0)$ \\
Q Wave & $(9.1)$ \\
QTc Prolongation & $(0)$ \\
\hline AV - Atrioventricular, ICH - Intracranial hemorrhag \\
\hline
\end{tabular}

In a review on cardiac injury following cerebral and subarachnoid hemorrhages, ECG abnormalities were found in more than $70 \%$ of patients who had no previous history of heart disease in 2 studies. ${ }^{13,17,18}$ Among SAH and $\mathrm{IPH}$, the most common abnormality seen was QTc prolongation (in 36-50\% of cases), followed by ST-segment changes (in 21.6-26\% of patients), and sometimes sinus bradycardia, tachycardia, or T-wave inversion was found. ${ }^{18-20}$ In our study, ST-segment changes were the most frequent ECG abnormalities following $\mathrm{ICH}$ in $32.7 \%$ of cases, followed by T-wave inversion in $16.3 \%$. Incidence of ECG abnormalities ranged from $33.3 \%$ in IPH to $37.5 \%$ in $\mathrm{SAH}$, with SDH patients exhibiting ST-segment changes in 36.4\% of cases. QTc prolongation was only observed in one patient.

ST-segment changes, T-wave inversion, and QTc prolongation are correlated in the literature with characteristics and outcomes of IPH. ${ }^{8,16,21}$ In our study, death was more common in patients who did not exhibit ST-segment changes (17.5\% vs $9.4 \%)$. The 2 patients with IPH for whom ECG records were available and who died were without ST-segment changes. However, the percentage of patients with ST-segment changes or T-wave inversion who had worse GCS or mRS scores 
was higher than the percentage from their counterparts with better scores. The one patient with an ECG record that had neurogenic pulmonary edema also had ST-segment changes. Consistently, ST-segment changes and sinus tachycardia were significantly associated with $\mathrm{IPH}, \mathrm{SAH}$, and spontaneous mixed $\mathrm{ICH}$ in children compared to other types of hemorrhage. The one patient who had QTc prolongation in our study had $\mathrm{IPH}$, but it was not significantly associated with other characteristics or outcomes.

Arrhythmias were the most studied in the literature and they correlated with SAH and its outcomes. ${ }^{22-24}$ Arrhythmias were assessed in our study but only included atrial fibrillation and sinus tachycardia, which could be a result of small sample sizes. One of the 3 patients with atrial fibrillation for whom ECG data were available died (33.3\%), but this was not statistically significant compared to patients without atrial fibrillation $(p=.384)$. Arrhythmias were associated with advanced age, ${ }^{25}$ and advanced age was correlated with abnormal findings in general; however, the association with arrhythmias, in particular, disappeared with age since patients with arrhythmias were distributed equally among the adult ICH groups. Other ECG changes and serum troponin levels are frequently associated with characteristics and poor outcomes of SAH. 5,26,27

Adult SDHs have been sparsely studied in relation to ECG changes, and most of the literature is based on cases of head trauma. Abnormalities associated with ischemia, such as ST-segment changes, T-wave inversions, and arrhythmias were observed only in a few cases. ${ }^{28-30}$ In our study, $72.4 \%$ of the ECG's were abnormal. Patients with SDH were the only ones with $\mathrm{Q}$ waves or left axis deviation or bundle branch blocks ( $3 \%$ and $21.2 \%$, respectively). An association between AV block and neurologic disease was also observed in these patients. However, no association with clinical outcomes was observed in our study, nor has it been mentioned in the literature when considered separately from other types of $\mathrm{ICH}$.

QTc prolongation is associated with IPH and vasospasm in $\mathrm{SAH},{ }^{31,32}$ while AV block is considered an arrhythmia that is potentially fatal. ${ }^{13}$ Interestingly, we observed that QTc prolongation and AV blocks were associated with neurological disease across all ICH types. Although our study had a small number of patients with neurological disease, it is found to be a significant risk factor for QTc prolongation. ${ }^{33} \mathrm{AV}$ block is commonly associated with Lyme disease, which has neurological components, ${ }^{34}$ and autoimmune disease, ${ }^{35}$ but its relation to other neurological disease has not been well-studied.

It is recommended that to reduce or predict the incidence of poor outcomes following ICH (and head trauma in general), ECG should be utilized alongside measures of serum cardiac troponins at the time of admission, followed by cardiac monitoring for arrhythmias for the first 2 to 3 days following hospitalization. ${ }^{1}$ These recommendations could play a role in the planning of surgical intervention. ${ }^{30,36-38}$ The utilization of the neurocardiac injury score may also be useful. ${ }^{39}$ It has been suggested that the cause of cardiac injury following neurological insult may be a result of sympathetic nervous system activation related to increased intracranial pressure and stress cardiomyopathy. ${ }^{6,40}$ The ECG changes in pediatric ICH cases have been reported in a few studies, mostly in trauma settings. ${ }^{41-43}$ These changes include arrhythmias, ST-segment abnormalities and QTc prolongation, though no correlation has been observed with clinical outcomes.

Limitations to our study included the retrospective design, which, unfortunately, may have provided less relevant data and incomplete ECG records, as ECG is not routinely performed for all ICH patients. The total number of participants is also limited especially when divided to subgroups or addressing ECG records; this limits the statistical power of our analysis. We also did not have complete outcome measures for all patients such as scores from the Glasgow Outcome Scale (GOS) or Modified Rankin Scale. This resulted in fewer ECG findings and possibly ambiguous associations.

Conclusion. ECG changes following ICH, with or without trauma, have been proposed as an important prognostic tool. The findings of our study are not strongly suggestive of a prognostic value of ECG in the context of ICH but, did show strong associations between $\mathrm{SDH}$ and 3rd degree AV block; therefore, ECG monitoring may be important in these cases, though further research is needed to assess its potential to predict and improve clinical outcomes following head trauma and all types of ICH in adults and children.

Acknowledgement. The authors would like to thank each of Shomokh F. Alotaibi, Raghad A. Alfakeeh, and Alya S. Alharbi for their valuable contribution in the data collection for this study. The authors would also like to thank Editage (www.editage.com) for English language editing.

\section{References}

1. Gurdjian ES, Webster JE. Traumatic intracranial hemorrhage. The American Journal of Surgery 1948; 75: 82-98.

2. Fischbein NJ, Wijman CA. Nontraumatic intracranial 
hemorrhage. Neuroimaging Clinics 2010; 20: 469-492.

3. McCormack RF, Hutson A. Can computed tomography angiography of the brain replace lumbar puncture in the evaluation of acute-onset headache after a negative noncontrast cranial computed tomography scan?. Acad Emerg Med 2010; 17: 444-445.

4. An SJ, Kim TJ, Yoon BW. Epidemiology, risk factors, and clinical features of intracerebral hemorrhage: an update. $J$ Stroke 2017; 19: 3-10.

5. Zhang L, Qi S. Electrocardiographic Abnormalities Predict Adverse Clinical Outcomes in Patients with Subarachnoid Hemorrhage. J Stroke Cerebrovasc Dis 2016; 25: 2653-2659.

6. Hasanin A, Zakaria D, Allam A. Cardiac injury in severe head trauma: a review of literature. J Neurol Neuromedicine 2016: 41-43.

7. Elsharkawy H, Abd-Elsayed A, El-Hadi S, Provencio J, Tetzlaff J. Fluctuating Electrocardiographic Changes Predict Poor Outcomes After Acute Subarachnoid Hemorrhage. Ochsner J 2016; 16: 225-229.

8. Hjalmarsson C, Bergfeldt L, Bokemark L, Manhem K, Andersson B. Electrocardiographic abnormalities and elevated cTNT at admission for intracerebral hemorrhage: predictors for survival? Ann Noninvasive Electrocardiol 2013; 18: 441-449.

9. Bhattacharya IS, Sandeman D, Dweck M, McKie S, Francis M. Electrocardiographic abnormalities in a patient with subarachnoid haemorrhage. Case Reports 2011; 2011: bcr0820103253.

10. Sakr YL, Ghosn I, Vincent JL. Cardiac manifestations after subarachnoid hemorrhage: A systematic review of the literature. Prog Cardiovasc Dis 2002; 45: 67-80.

11. Chatterjee S. ECG Changes in Subarachnoid Haemorrhage: A Synopsis. Neth Heart J 2011; 19: 31-34.

12. Qaqa AY, Suleiman A, Alsumrain M, Debari VA, Kirmani J, Shamoon FE. Electrocardiographic abnormalities in patients presenting with intracranial parenchymal haemorrhage. Acta Cardiol. 2012; 67: 635-639.

13. Pinnamaneni S, Aronow WS, Frishman WH. Neurocardiac injury after cerebral and subarachnoid hemorrhages. Cardiology in review 2017; 25: 89-95.

14. Naredi S, Lambert G, Eden E, Zall S, Runnerstam M, Rydenhag B, et al. Increased sympathetic nervous activity in patients with nontraumatic subarachnoid hemorrhage. Stroke 2000; 31: 901-906.

15. Masuda T, Sato K, Yamamoto S, Matsuyama N, Shimohama T, Matsunaga A, et al. Sympathetic nervous activity and myocardial damage immediately after subarachnoid hemorrhage in a unique animal model. Stroke 2002; 33: 1671-1676.

16. Takeuchi S, Nagatani K, Otani N, Wada K, Mori K. Electrocardiograph abnormalities in intracerebral hemorrhage. Journal of Clinical Neuroscience 2015; 22: 1959-1962.

17. Daniele O, Caravaglios G, Fierro B, Natalè E. Stroke and cardiac arrhythmias. J Stroke Cerebrovasc Dis 2002; 11: 28-33.

18. van Bree MD, Roos YB, van der Bilt IA, Wilde AA, Sprengers $\mathrm{ME}$, de Gans K, et al. Prevalence and characterization of ECG abnormalities after intracerebral hemorrhage. Neurocrit Care 2010; 12: 50-55.

19. Lleva P, Aronow WS, Amin H, Sandhu R, D'Aquila K. Prevalence of electrocardiographic abnormalities in patients with ischemic stroke, intracerebral hemorrhage, and subarachnoid hemorrhage. Archives of Medical Science 2008; 4: 259 .
20. Popescu D, Cristina LA, Mergeani A, Bajenaru OA, Antochi FA. Lead electrocardiogram changes after supratentorial intracerebral hemorrhage. Maedica (Buchar) 2012; 7: 290-294.

21. Christensen H, Christensen AF, Boysen G. Abnormalities on ECG and telemetry predict stroke outcome at 3 months. $J$ Neurol Sci. 2005; 234: 99-103.

22. Frontera JA, Parra A, Shimbo D, FernandezA, Schmidt JM, Peter P, et al Cardiac arrhythmias after subarachnoid hemorrhage: risk factors and impact on outcome. Cerebrovascular diseases 2008; 26: 71-78.

23. Frangiskakis JM, Hravnak M, Crago EA, Tanabe M, Kip KE, Gorcsan J, et al. Ventricular arrhythmia risk after subarachnoid hemorrhage. Neurocritical care 2009; 10: 287-294.

24. Schmidt JM, Crimmins M, Lantigua H, Fernandez A, Zammit $\mathrm{C}$, Falo C, et al. Prolonged elevated heart rate is a risk factor for adverse cardiac events and poor outcome after subarachnoid hemorrhage. Neurocritical care 2014; 20: 390-398.

25. Kallmünzer B, Breuer L, Kahl N, Bobinger T, Raaz-Schrauder D, Huttner HB, et al. Serious cardiac arrhythmias after stroke: incidence, time course, and predictors-a systematic, prospective analysis. Stroke 2012; 43: 2892-2897.

26. Van der Bilt IA, Hasan D, Vandertop WP, Wilde AA, Algra A, Visser FC, et al. Impact of cardiac complications on outcome after aneurysmal subarachnoid hemorrhage: a meta-analysis. Neurology 2009; 72: 635-642.

27. Yousef KM, Crago E, Lagattuta TF, Hravnak M. Clinical Presentation to the Emergency Department Predicts Subarachnoid Hemorrhage-Associated Myocardial Injury. $J$ Emerg Nurs 2018; 44: 132-138.

28. Iltumur K, Tamam Y, Karahan Z, Guzel A, Altındag R. Coexisting subarachnoid hemorrhage and subdural hematoma mimicking acute anterolateral myocardial infarction. J Electrocardiol 2007; 40: 422-425.

29. Póvoa R, Cavichio L, Almeida AL, Viotti D, Ferreira C, Galvão $\mathrm{L}$, et al. Electrocardiographic abnormalities in neurological diseases. Arq Bras Cardiol 2003; 80: 351-358.

30. Hashemian AM, Ahmadi K, Taherinia A, Sharifi MD, Ramezani J, Jazayeri SB, et al. ECG changes of cardiac origin in elderly patients with traumatic brain injury. Medical journal of the Islamic Republic of Iran 2015; 29: 306.

31. Ibrahim GM, Macdonald RL. Electrocardiographic changes predict angiographic vasospasm after aneurysmal subarachnoid hemorrhage. Stroke 2012; 43: 2102-2107.

32. Lederman YS, Balucani C, Lazar J, Steinberg L, Gugger J, Levine SR. Relationship between QT interval dispersion in acute stroke and stroke prognosis: a systematic review. J Stroke Cerebrovasc Dis 2014; 23: 2467-2478.

33. Vandael E, Vandenberk B, Vandenberghe J, Willems R, Foulon $\mathrm{V}$. Risk factors for QTc-prolongation: systematic review of the evidence. Int J Clin Pharm 2017; 39: 16-25.

34. Wan D, Blakely C, Branscombe P, Suarez-Fuster L, Glover B, Baranchuk A. Lyme carditis and high-degree atrioventricular block. Am J Cardiol 2018; 121: 1102-1104.

35. Cimaz R, Lehman TJ, editors. Pediatrics in systemic autoimmune diseases. Amsterdam (NL): Elsevier; 2016.

36. Jain A, Dogra N, Mangal K. To evaluate the heart or not in emergency neurosurgical head-injured patients with ST elevation. Ann Card Anaesth 2010; 13: 268-269.

37. Naidech AM, Kreiter KT, Janjua N, Ostapkovich ND, Parra A, Commichau C, et al. Cardiac troponin elevation, cardiovascular morbidity, and outcome after subarachnoid hemorrhage. Circulation 2005; 112: 2851-2856. 
38. Poldermans D, Bax JJ, Boersma E, De Hert S, Eeckhout E, Fowkes G, et al. Guidelines for pre-operative cardiac risk assessment and perioperative cardiac management in non-cardiac surgery: the Task Force for Preoperative Cardiac Risk Assessment and Perioperative Cardiac Management in Non-cardiac Surgery of the European Society of Cardiology (ESC) and endorsed by the European Society of Anaesthesiology (ESA). European Journal of Anaesthesiology (EJA) 2010; 27: 92-137.

39. Hasanin A, Kamal A, Amin S, Zakaria D, El Sayed R, Mahmoud K, et al. Incidence and outcome of cardiac injury in patients with severe head trauma. Scand J Trauma Resusc Emerg Med 2016; 24: 58.
40. Bhagat H, Narang R, Sharma D, Dash HH, Chauhan H. ST elevation-An indication of reversible neurogenic myocardial dysfunction in patients with head injury. Ann Card Anaesth 2009; 12: 149-151.

41. Dash M, Bithal PK, Prabhakar H, Chouhan RS, Mohanty B. ECG changes in pediatric patients with severe head injury. $J$ Neurosurg Anesthesiol 2003; 15: 270-273.

42. Bourdages M, Bigras JL, Farrell CA, Hutchison JS, Lacroix J. Cardiac arrhythmias associated with severe traumatic brain injury and hypothermia therapy. Pediatric Critical Care Medicine 2010; 11: 408-4014.

43. Ozdemir D, Ozdemir N, Unal N, Tektas S. QTc dispersion in children with severe head trauma. Pediatric emergency care 2005; 21: 658-661.

\section{References}

* References should be primary source and numbered in the order in which they appear in the text. At the end of the article the full list of references should follow the Vancouver style.

* Unpublished data and personal communications should be cited only in the text, not as a formal reference.

* The author is responsible for the accuracy and completeness of references and for their correct textual citation.

* When a citation is referred to in the text by name, the accompanying reference must be from the original source.

* Upon acceptance of a paper all authors must be able to provide the full paper for each reference cited upon request at any time up to publication.

* Only 1-2 up to date references should be used for each particular point in the text.

Sample references are available from:

http://www.nlm.nih.gov/bsd/uniform_requirements.html 